\title{
Überprüfung bezüglich Erdbeben von Holzbalkendecken in Bestandesbauten
}

\section{Journal Article}

Author(s):

Wenk, Thomas (1); Brunner, Roland; Almeida, João P.; Beyer, Katrin

Publication date:

2020-04

Permanent link:

https://doi.org/10.3929/ethz-b-000413418

Rights / license:

In Copyright - Non-Commercial Use Permitted

Originally published in:

D-A-CH-Mitteilungsblatt [enthalten in: Bauingenieur] 95(4) 


\section{Überprüfung bezüglich Erdbeben von Holzbalkendecken in Bestandesbauten}

T. Wenk, R. Brunner, J. Pacheco de Almeida, K. Beyer
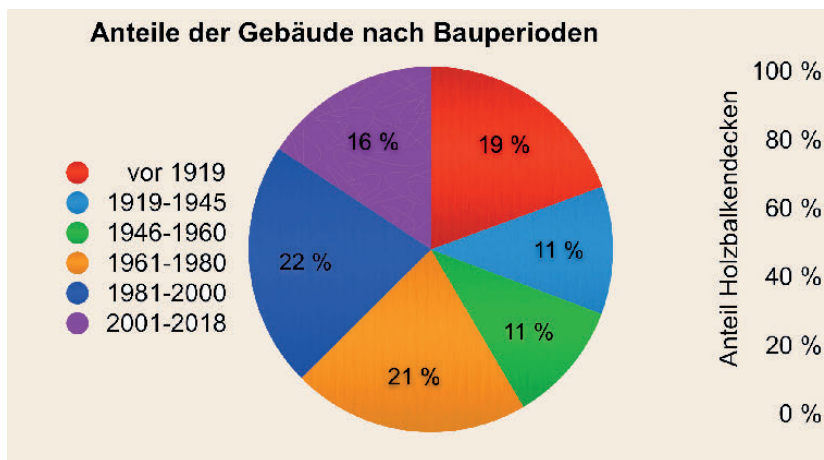

Anteile der Gebäude mit Holzbalkendecken nach Bauperioden

Bild 1. Anteile der Gebäude in der Schweiz nach Bauperioden (links) und geschätzte Anteile der Gebäude mit Holzbalkendecken nach Bauperioden (rechts) Abb.:T. Wenk

\section{Einleitung}

Viele bestehende Mauerwerksgebäude in der Schweiz weisen Holzbalkendecken als Deckenkonstruktion auf. Dies triff vor allem für Gebäude zu, die bis in die erste Hälfte des 20. Jahrhundert erbaut wurden. Holzbalkendecken sind in ihrer Ebene sehr flexibel und in den Wänden meist ungenügend verankert, da Fassadenanker und Ringbalken in der Schweiz weitgehend fehlen. Dies kann zu einem ungünstigen Verhalten während eines Erdbebens führen, sowohl bezüglich globalem Verhalten des Gebäudes mit Beanspruchung der Wände in ihrer Ebene als auch bezüglich lokalem Verhalten der Mauerwerkswände quer zur Wandebene.

Bei den bisher üblichen kraftbasierten Überprüfungen von bestehenden Mauerwerksgebäuden mit ursprünglich für die Bemessung von Neubauten entwickelten, relativ konservativen Berechnungsverfahren resultiert in der Regel eine zu niedrige Erdbebensicherheit, so dass eine Ertüchtigung der Wände, der Decken und deren Verankerung erforderlich wird. Bei genauerer Untersuchung mit modernen verformungsbasierten Verfahren zeigt sich, dass für niedrige bis mittlere Seismizität - wie in den D-A-CHLändern vorherrschend - oft der Ist-Zustand mit minimaler Ertüchtigung oder sogar ganz ohne Ertüchtigung akzeptiert werden könnte, falls über Reibung horizontale Verankerungskräfte von den Holzbalken auf die Mauerwerkswände übertragen werden könnten. Rütteltischversuche an zwei zusammengebauten, $8 \mathrm{~m}$ hohen Basler Altstadthäusern aus Natursteinmauerwerk mit Holzbalkendecken im Massstab $1: 2$ bestätigten diesen Sachverhalt für das globale und lokale Erdbebenverhalten [1].

In diesem Beitrag werden Reibungsbeiwerte für verschiedene Verbindungsarten zwischen Holzbalken und Mauerwerkswänden basierend auf statisch-zyklischen Reibungsversuchen präsentiert, die in der Praxis für die Überprüfung von Gebäuden mit Holzbalkendecken verwendet werden können.

\section{Bedeutung der Holzbalkendecken}

Zur Bestimmung der Bedeutung der Holzbalkendecken in Bestandesbauten werden die im Jahre 2018 vorhandenen 1,7 Mio. Gebäude mit Wohnnutzung in der Schweiz in sechs Bauperioden eingeteilt (Bild 1), für jede davon wird ein relativer Anteil mit Holzbalkendecken grob abgeschätzt und anschliessend werden die einzelnen Anteile zusammengezählt. So kann berücksichtigt werden, dass in früheren Bauperioden bei einem wesentlich grösseren Anteil der Gebäude Holzbalkendecken eingebaut wurden als in neuerer Zeit. Da die Gebäudedaten des Bundesamts für Statistik keinerlei Angaben zu den Bauweisen der Decken umfassen, wird dieser indirekte Weg beschritten, um aus der zeitlichen Gliederung der Baujahre des Gesamtbestands der Gebäude den Anteil der Holzbalkendecken abzuschätzen [2].

Die betrachteten sechs Bauperioden lassen sich bezüglich Deckenbauweisen folgendermaßen charakterisieren (Bild 1):

- Vor 1919: Holzbalkendecken sind die verbreitete Bauweise der Decken der oberen Geschosse im Wohnungsbau. Für die Bodendecke des Erdgeschosses werden oft Gewölbedecken verwendet. Im Industrie- und Verwaltungsbau werden Stahlbetondecken und Deckenkonstruktionen mit Stahlträgern eingeführt. Der Anteil der Gebäude mit Holzbalkendecken wird auf $80 \%$ geschätzt.

- 1919-1945: Der Anteil der Holzbalkendecken geht zurück. Sie bleiben jedoch die wichtigste Bauweise der Decken im Wohnungsbau. Vermehrt werden Hourdisdecken, vor allem im Nassbereich der Wohnungen, eingesetzt. Der Anteil der Gebäude mit Holzbalkendecken wird auf $60 \%$ geschätzt.

- 1945-1960: Nach dem 2. Weltkrieg werden die Holzbalkendecken im Wohnungsbau vor allem aus Komfortgründen bezüglich Schallschutz und Deckenschwingungen mehr und mehr durch Stahlbetondecken ersetzt. Ferner wird der Brandschutz der Holzbalkendecken bei mehrgeschossigen Bauten als ungenügend eingestuft. Der Anteil der Gebäude mit Holzbalkendecken fällt auf etwa $30 \%$. 
- 1961-1980: Der Ersatz der Holzbalkendecken durch Stahlbetondecken beschleunigt sich, da Wohnungen mit Holzbalkendecken sowohl von Mietern als auch von Eigentümern als veraltet bewertet werden. Der verbleibende Anteil von Gebäuden mit Holzbalkendecken wird auf $10 \%$ geschätzt.

- 1981-2000: Der bisherige Trend des Ersatzes der Holzbalkendecken setzt sich fort. Der Restanteil wird auf $5 \%$ geschätzt.

- 2000-2018: Bei den Neubauten aus der letzten Bauperiode wird angenommen, dass sie eine akzeptable Erdbebensicherheit aufweisen. Der Anteil der Holzbalkendecken wird auf $5 \%$ geschätzt wie in der vorhergehenden Bauperiode.

Zusammengerechnet ergibt sich ein Anteil der Holzbalkendecken von $28 \%$ am heutigen Gesamtbestand von 1,7 Mio. Gebäuden mit Wohnnutzung, das heißt ein gutes Viertel sollte zumindest bezüglich Erdeben überprüft werden und, falls erforderlich, ertüchtigt werden.

\section{Holzbalkendecken unter Erdbebeneinwirkung}

Bei einem Mauerwerksgebäude mit Holzbalkendecken greifen die Erdbebenkräfte primär in den Wänden an, da die Wände eine große Masse im Vergleich zu den leichten Decken aufweisen. Bild 2 veranschaulicht schematisch den Verformungszustand und den Kraftfluss unter Erdbebenbeanspruchung in Längsrichtung, das heißt parallel zur Balkenlage und zu den Fassadenwänden, für den Fall, dass keine Fassadenanker vorhanden sind. Die relativ großen Trägheitskräfte infolge der Fassadenwandmassen werden direkt von der Fassadenwand abgetragen (breite blaue Pfeile im Bild 2 rechts). Die kleinen Trägheitskräfte aus den bescheidenen Deckenmassen (durchsichtige blaue Pfeile) werden durch die Decken auf die Fassadenwände übertragen. $\mathrm{Zu}$ diesem Zweck müssen die Decken eine gewisse Steifigkeit und Festigkeit in der Deckenebene aufweisen. Die Trägheitskräfte infolge der Giebelwandmassen bewirken eine Beanspruchung quer zur Wandebene der Giebelwand (rote Pfeile). Die senkrecht zur Giebelwand laufenden Holzbalken übernehmen über Reibung Horizontalkräfte, die stabilisierend auf die Giebelwände wirken (rote Pfeile im Bild 2 links). Diese Horizontalkräfte müssen ebenfalls über die Decken in die Fassadenwände geleitet werden (rote Pfeile). Ohne diese Reibungskräfte würde die Giebelwand, abgesehen von der Stabilisierung über die gemauerten Eckverbindung zu den Fassadenwänden, als freistehender Kragarm über die ganze Gebäudehöhe beansprucht. Die Deckenbeanspruchung aus den horizontalen Erdbebenkräften bleibt im Allgemeinen gering, falls das Gebäude regelmässig im Grundriss und im Aufriss ausgelegt ist.

\section{Konstruktionsarten von Holzbalkendecken}

Die unterschiedlichen Konstruktionsarten von Holzbalkendecken in den letzten Jahrhunderten in der Schweiz können vereinfachend auf je eine Bauperiode vor und nach 1900 aufgeteilt werden. Tabelle 1 fasst die Konstruktionsarten und deren charakteristischen Parameterbereiche, soweit für die Reibungskräfte zwischen Holzbalken und Mauerwerkswänden relevant, grob aufgeteilt auf die beiden Bauperioden zusammen. Die resultierenden vertikalen Balkenreaktionskräfte auf die Wände bleiben für beide Bauperioden im gleichen Bereich von etwa $0,6 \mathrm{kN}$ bis $5 \mathrm{kN}$, wobei der Balkenquerdruck über die Einbindungslänge in der Wand

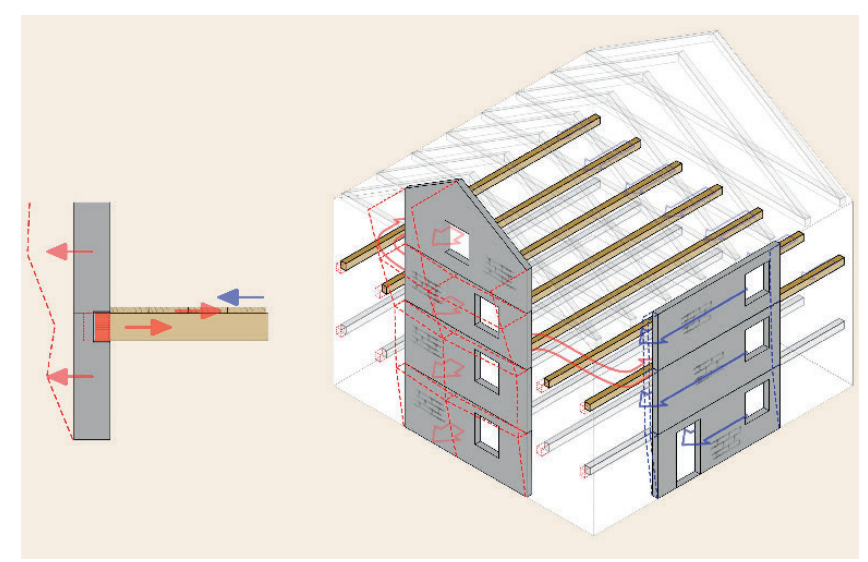

Bild 2. Mauerwerksgebäude mit Holzbalkendecken unter Erdbebeneinwirkung in Längsrichtung (rechts), Detail eines Holzbalkenauflagers in der Giebelwand (links) Abb.: R. Brunner

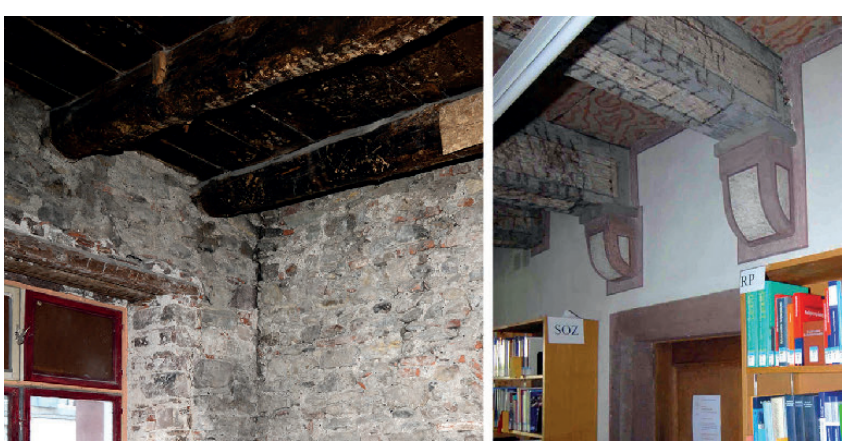

Bild 3. Holzbalken-Wand-Verbindung in mittelalterlichen Gebäuden in Basel: Direkte Einbettung der Holzbalken in der Wand (links) und Lagerung auf Steinkonsolen (rechts) Abb.: T. Wenk

in der neueren Bauperiode mit bis zu $500 \mathrm{kN} / \mathrm{m}^{2}$ stark zugenommen hat.

Bei der Bauperiode vor 1900 ist die direkte Einbettung der Holzbalken in der Wand der häufigste Fall der Verbindung (Bild 3, links). Die Einbindungslänge liegt typischerweise zwischen 0,1 $\mathrm{m}$ und 0,2 $\mathrm{m}$ oder erreicht die halbe Wanddicke (Tabelle 1). Doch trifft man auch auf indirekte Lagerung der Holzbalken, nämlich der Lagerung auf Steinkonsolen in der Wand (Bild 3, rechts) oder der Lagerung auf Streichbalken. Diese laufen entlang der Wand und sind auf Steinkonsolen, die aus der Wand ragen, gelagert (Bild 4). Die indirekte Lagerung auf Streichbalken wird häufig in Kellergeschossen zum Schutz der Holzbalken vor der Mauerwerksfeuchte verwendet. Eine weitere Variante ist die Lagerung auf Wandvorsprüngen, die sich typischerweise durch eine geschossweise Reduktion der Wanddicke nach oben im Gebäude ergeben. Die Holzbalken werden entweder direkt auf den Wandvorsprung oder indirekt auf eine Holzschwelle gelegt, die wiederum dem Wandvorsprung entlang verläuft.

In der Bauperiode nach 1900 findet man als Lagerungsart der Holzbalken praktisch nur die direkte Einbettung in der Wand. Ein typisches Lagerungsdetail eines Gebäudes von 1960 zeigt Bild 5. Beim kürzlich erfolgten Rückbau, der die Holzbalkenverankerung freilegte, ist im Bildausschnitt rechts zu erkennen, dass der Holzbalken über die halbe Wanddicke des unteren Geschosses mit einer Dicke von $25 \mathrm{~cm}$ eingebunden ist.

Für beide Bauperioden ist typisch, dass in der Regel weder Fassadenanker noch sonstige mechanische Anker vorhanden sind. 
Tabelle 1. Konstruktionsarten von Holzbalkendecken in der Schweiz mit charakteristischen Parameterbereichen aufgeteilt auf zwei Bauperioden vor und nach 1900

\begin{tabular}{|c|c|c|}
\hline & Mittelalter bis 1900 & seit 1900 \\
\hline Holzart & $\begin{array}{l}\text { Balken: Fichte oder Kiefer } \\
\text { Hauptträger: Fichte oder Eiche }\end{array}$ & $\begin{array}{l}\text { Balken: Fichte oder Kiefer } \\
\text { keine Hauptträger }\end{array}$ \\
\hline Balkenspannweite I & $I \approx 3 \mathrm{~m}$ bis $4 \mathrm{~m}$ & $I \approx 4 \mathrm{~m}$ bis $6 \mathrm{~m}$ \\
\hline Achsabstand der Balken $e$ & $e \approx 1 \mathrm{~m}$ & $\begin{array}{c}e \approx 0,6 \mathrm{~m} \\
\text { (Die } 0,6 \mathrm{~m} \text { sind noch heute eine Zimmermannsregel) }\end{array}$ \\
\hline $\begin{array}{l}\text { Balkenquerschnitt: } \\
h \text { : Höhe; } b \text { : Breite; } \\
\text { l: Spannweite }\end{array}$ & $\begin{array}{l}b \text { von } 0,15 \mathrm{~m} \text { bis } 0,3 \mathrm{~m} \\
h \text { von } 0,15 \mathrm{~m} \text { bis } 0,3 \mathrm{~m}\end{array}$ & $\begin{array}{l}\qquad h / b \approx 2 \text { und } l / h \approx 20 \\
\text { (Diese Werte sind noch heute Zimmermannsregeln) }\end{array}$ \\
\hline Oberflächenbehandlung & gehackt & $\begin{array}{l}\text { gesägt unbehandelt } \\
\text { oder gehobelt }\end{array}$ \\
\hline Verbindungsart mit Wand & $\begin{array}{l}\text { Einbindung, Wandvorsprünge } \\
\text { Steinkonsolen, Streichbalken }\end{array}$ & $\begin{array}{l}\text { Einbindung, } \\
\text { Wandvorsprünge }\end{array}$ \\
\hline Einbindungslänge in Wand $l_{e}$ & $\begin{array}{l}\qquad 0,1 \mathrm{~m} \leq I_{e} \leq 0,2 \mathrm{~m} \\
\text { oder halbe Wanddicke }\end{array}$ & $0,1 \mathrm{~m} \leq I_{e} \leq 0,15 \mathrm{~m}$ \\
\hline Deckenaufbau & $\begin{array}{c}50 \mathrm{~mm} \text { bis } 80 \mathrm{~mm} \text { dicke Fichte- oder Kieferbretter auf Balkenlage, } \\
\text { unten: offen oder unterschiedliche Schüttungen }\end{array}$ & $\begin{array}{c}25 \mathrm{~mm} \text { bis } 35 \mathrm{~mm} \text { dicke Fichte- oder Kieferbretter auf } \\
\text { Balkenlage, unten: Gips-Binsen-Matte }\end{array}$ \\
\hline Eigengewicht der Decke & $\begin{array}{l}\text { ohne Schüttung: } 0,4 \text { bis } 0,6 \mathrm{kN} / \mathrm{m}^{2} \\
\text { mit Schüttung: } 0,6 \text { bis } 2,0 \mathrm{kN} / \mathrm{m}^{2}\end{array}$ & $\begin{array}{l}\text { ohne Schüttung: } 0,5 \text { bis } 1,0 \mathrm{kN} / \mathrm{m}^{2} \\
\text { mit Schüttung: } 1,0 \text { bis } 2,0 \mathrm{kN} / \mathrm{m}^{2}\end{array}$ \\
\hline Nutzlast auf Decke & $\leq 0,3 \times 2 \mathrm{kN} / \mathrm{m}^{2}=0,6 \mathrm{kN} / \mathrm{m}^{2}$ & $\leq 0,3 \times 2 \mathrm{kN} / \mathrm{m}^{2}=0,6 \mathrm{kN} / \mathrm{m}^{2}$ \\
\hline $\begin{array}{l}\text { Vertikale Balkenreaktionskraft } \\
\text { auf Wand ohne Lastverteilung } \\
\text { zwischen Balken }\end{array}$ & 0,6 bis $5,2 \mathrm{kN}$ & 0,6 bis $4,7 \mathrm{kN}$ \\
\hline $\begin{array}{l}\text { Balkenquerdruck über } \\
\text { Einbindungslänge } I_{e}\end{array}$ & $20 \mathrm{bis} 350 \mathrm{kN} / \mathrm{m}^{2}$ & 30 bis $500 \mathrm{kN} / \mathrm{m}^{2}$ \\
\hline
\end{tabular}
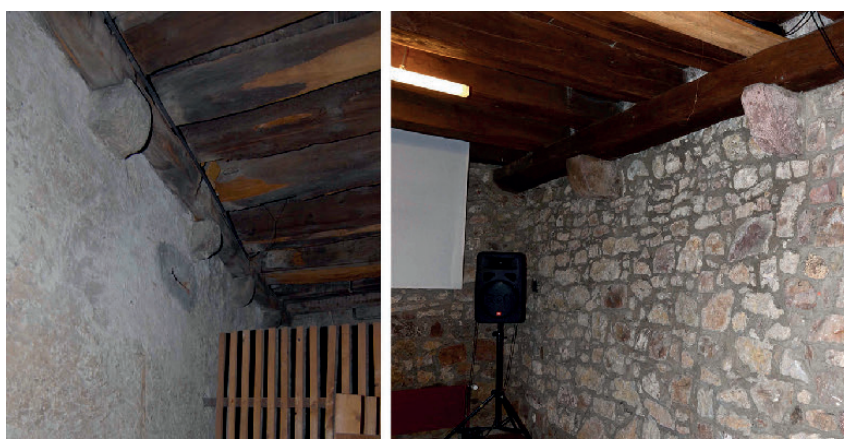

Bild 4. Streichbalken zur indirekten Lagerung der Holzbalken in mittelalterlichen Gebäuden in Basel Abb.:T. Wenk

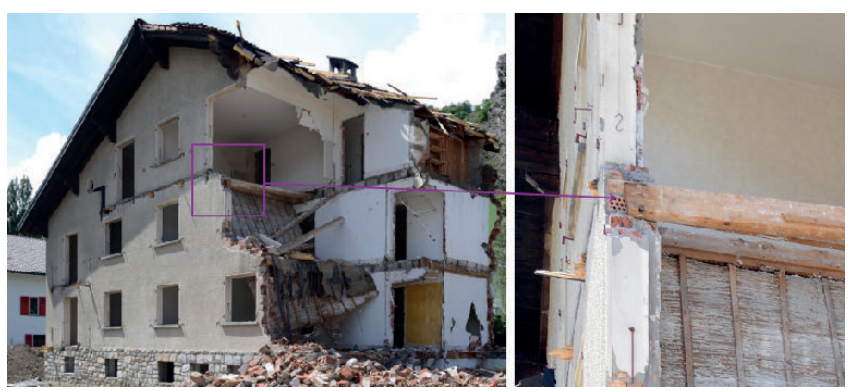

Bild 5. Beim Rückbau eines Gebäudes von 1960 (links) wird die typische Lagerung der Holzbalken mittels Einbettung über die halbe Dicke der Mauerwerkswand im Geschoss unterhalb des Holzbalkens sichtbar (rechts). Abb.: T. Wenk
Die Übertragung horizontaler Zugkräfte vom Holzbalken in die Wand kann somit fast nur über Reibung zusammen mit etwas Kohäsion erfolgen. Auf Druck können zusätzlich kleinere Kräfte von der Stirnfläche des Holzbalkens auf den äußeren Teil der Wand übertragen werden. Senkrecht zum Holzbalken erlaubt die Einbettung in die Wand eine gute Kraftübertragung horizontaler Querkräfte mittels Querdruck über die Einbindungslänge.

\section{Fassadenanker}

Fassadenanker sind in der Schweiz bei mittelalterlichen Gebäuden nur vereinzelt und bei neueren Gebäuden praktisch überhaupt nicht vorhanden. Bild 6 zeigt zwei seltene Beispiele von Mauerwerksgebäuden in der Basler Altstadt mit Fassadenankern. Das Erdbebenverhalten von Gebäuden mit Fassadenankern ist im Allgemeinen wesentlich besser im Vergleich zu Gebäuden ohne Fassadenanker, was auch beim Erdbeben von Amatrice 2016 in Italien bestätigt wurde. Dass Fassadenanker bei schweren Erdbeben stark beansprucht werden können, zeigt als eher seltenes Schadenbild Bild 7 das lokale Durchstanzversagen eines Fassadenankers beim Erdbeben von Amatrice. Auch weitere Konstruktionsdetails zur Verbesserung der Erdbebensicherheit von Mauerwerksbauten, wie Ringbalken in der Bodenebene des Dachgeschosses, blieben in der Schweiz weitgehend unbekannt, während sie in Italien schon seit Jahrhunderten verwendet werden.

Es ist unbestritten, dass Holzbalkendecken ohne mechanische Verankerungen in den Wänden für hochseismische Gebiete unge- 


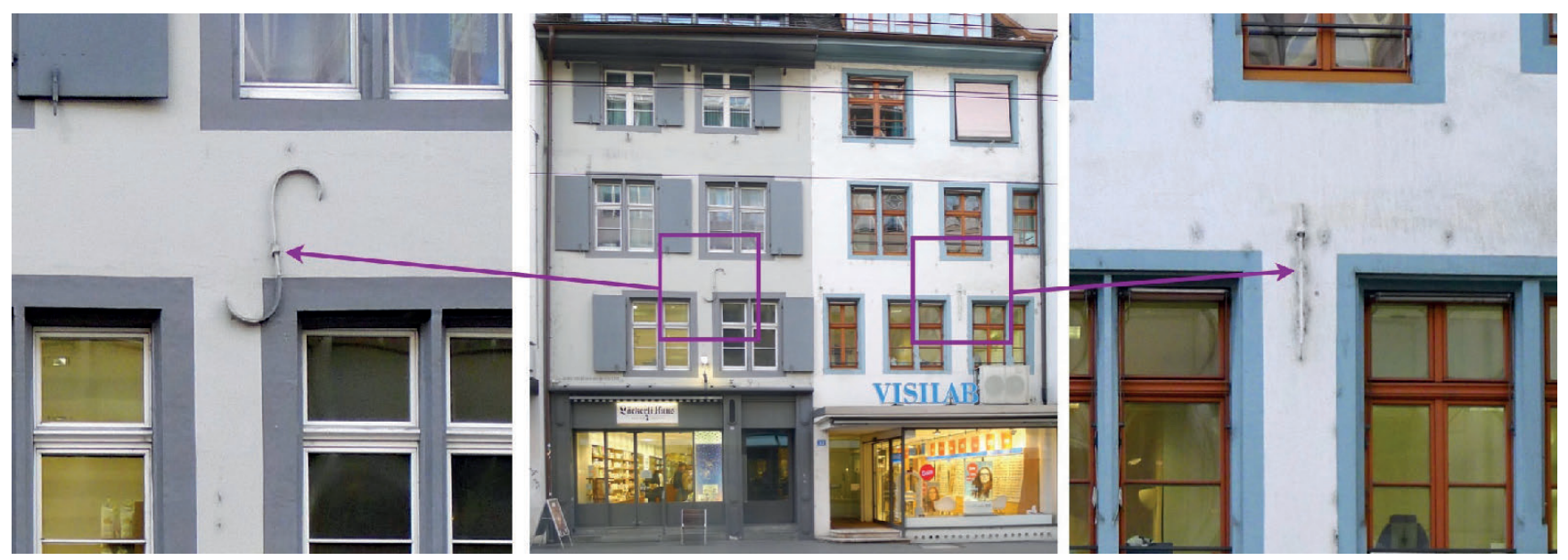

Bild 6. Fassadenanker in den Längswänden von zwei Mauerwerksgebäuden in der Basler Altstadt Abb.: T. Wenk

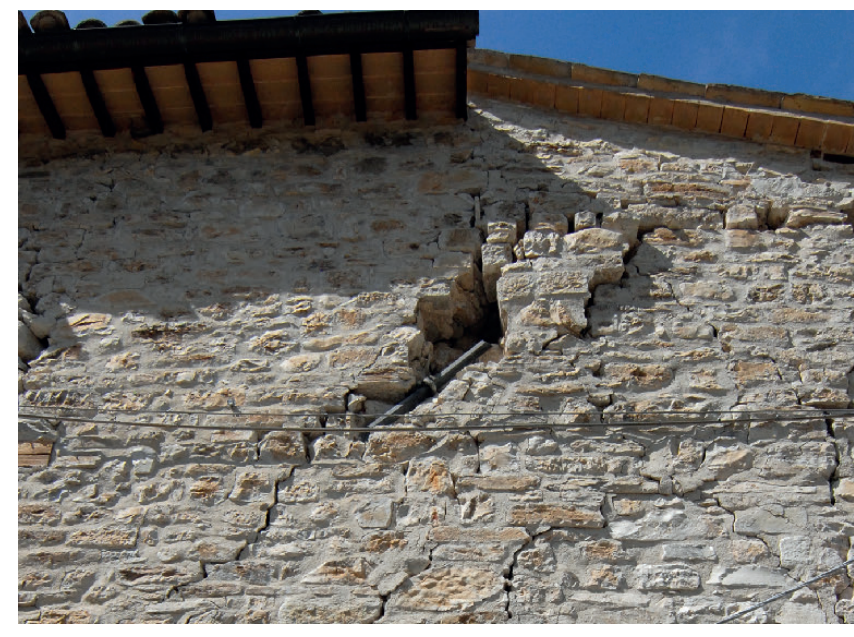

Bild 7. Durchstanzversagen eines Fassadenankers in Castelsantangelo sul Nera beim Erdbeben von Amatrice in Italien 2016 Abb.: T. Wenk

eignet sind. Andererseits belegen Untersuchungen historischer Erdbeben in der Schweiz, dass viele Gebäude mit unverankerten Holzbalkendecken auch stärkere Erdbeben überlebt haben, ohne dass heute Spuren von Schäden oder Reparaturen zu erkennen sind [3]. Werden solche Gebäude nach heutigen Normen überprüft [4], resultiert ohne Berücksichtigung der Reibung zwischen Holzbalken und Wand praktisch immer ein Ertüchtigungsbedarf. Mit Berücksichtigung der Reibung könnten zumindest ein größerer Teil dieser Gebäude in den Zonen niedriger Seismizität im Ist-Zustand ohne Ertüchtigung als genügend erdbebensicher akzeptiert werden, soweit die Regularitätskriterien im Grundriss und im Aufriss eingehalten sind.

\section{Zyklische Reibungsversuche}

Zur Bestimmung der Reibungsbeiwerte für die typischen Kontaktflächen von Holzbalkendecken, nämlich Holz auf Holz und Holz auf Mörtel, wurden zyklische Reibungsversuche an sogenannten „Triplet“-Versuchskörpern durchgeführt [5], [6]. Ähnliche Versuche wurden bereits zur Bestimmung der Reibungsbeiwerte zwischen OSB-Platten und Brettschichtholzplatten durchgeführt [7]. Bild 8 zeigt die Testmaschine für die Reibungsversuche Holz auf Mörtel mit dem eingebauten „Triplet“-Versuchskör- per bestehend aus einem zentralen Holzblock $175 \mathrm{~mm} \times 70 \mathrm{~mm}$, der zwischen zwei seitlichen Mörtelblöcken in vertikaler Richtung hin- und herbewegt wird. Die zyklische Reibungskraft wird durch die vertikale Presse erzeugt, während die konstant gehaltene Druckkraft durch die horizontale Presse auf den Versuchskörper aufgebracht wird.

Insgesamt wurden 187 Versuche durchgeführt [5]. Davon fallen je etwa die Hälfte auf Holz-Holz- und auf Holz-Mörtel-Versuchskörper. Weitere Versuchsparameter waren die Größe des Kontaktdrucks auf die Reibungsflächen sowie das Alter und die Oberflächenbehandlung des Holzes, wobei beim antiken Eichenholz eine gehackte Oberfläche verwendet wurde, während beim modernen Fichtenholz sowohl eine unbehandelte gesägte Oberfläche als auch eine gehobelte Oberfläche zum Einsatz kamen. Als Mörtel wurde ein hydraulischer Kalkmörtel im Kontakt mit dem antiken Eichenholz und ein moderner Zementmörtel im Kontakt mit dem modernen Fichtenholz verwendet.

Eine typische Kraft-Verschiebungs-Hysteresekurve eines Versuches mit einem Versuchskörper aus modernem gehobeltem Holz mit Mörtel ist in Bild 9 dargestellt. Die aufgebrachte Druckkraft von $2 \mathrm{kN}$ entspricht einem Kontaktdruck von 0,168 MPa. Dieser Wert liegt im mittleren Bereich des typischen Balkenquerdrucks, wie er in Tabelle 1 für die Bauperiode nach 1900 angegeben ist. Aus dem niedrigsten Wert der Gleitreibungskraft entlang des flachen Verlaufs der Hysteresekurve in Bild 8 von $2,3 \mathrm{kN}$ kann zusammen mit der Druckkraft von $2 \mathrm{kN}$ ein Gleitreibungsbeiwert von $\mu=2,3 /(2 \times 2)=0,6$ berechnet werden. Der zusätzliche Faktor zwei im Nenner ergibt sich dadurch, dass im „Triplet“-Versuche jeweils zwei Reibungsflächen zusammen aktiviert werden.

Eine Zusammenfassung der gemessenen Gleitreibungsbeiwerte für verschiedene Oberflächenbedingungen findet sich in Tabelle 2. Neben Mittelwert und Standardabweichung ist jeweils auch der untere charakteristische Wert gemäss Tragwerksnormen angegeben [8]. Er entspricht dem 5\%-Fraktilwert, der unter Annahme einer Normalverteilung der Versuchsresultate berechnet wurde. Die Mittelwerte variieren je nach Oberflächenbedingung zwischen 0,3 und 0,8 . Auffallend sind die niedrigen Werte für gehobeltes modernes Holz aufeinander, die etwa die Hälfte der Werte für die übrigen Oberflächenbedingungen betragen. Für alle untersuchten Holzarten liegen die Gleitreibungsbeiwerte im Kontakt mit Mörtel signifikant höher als im Kontakt untereinander. Dage- 


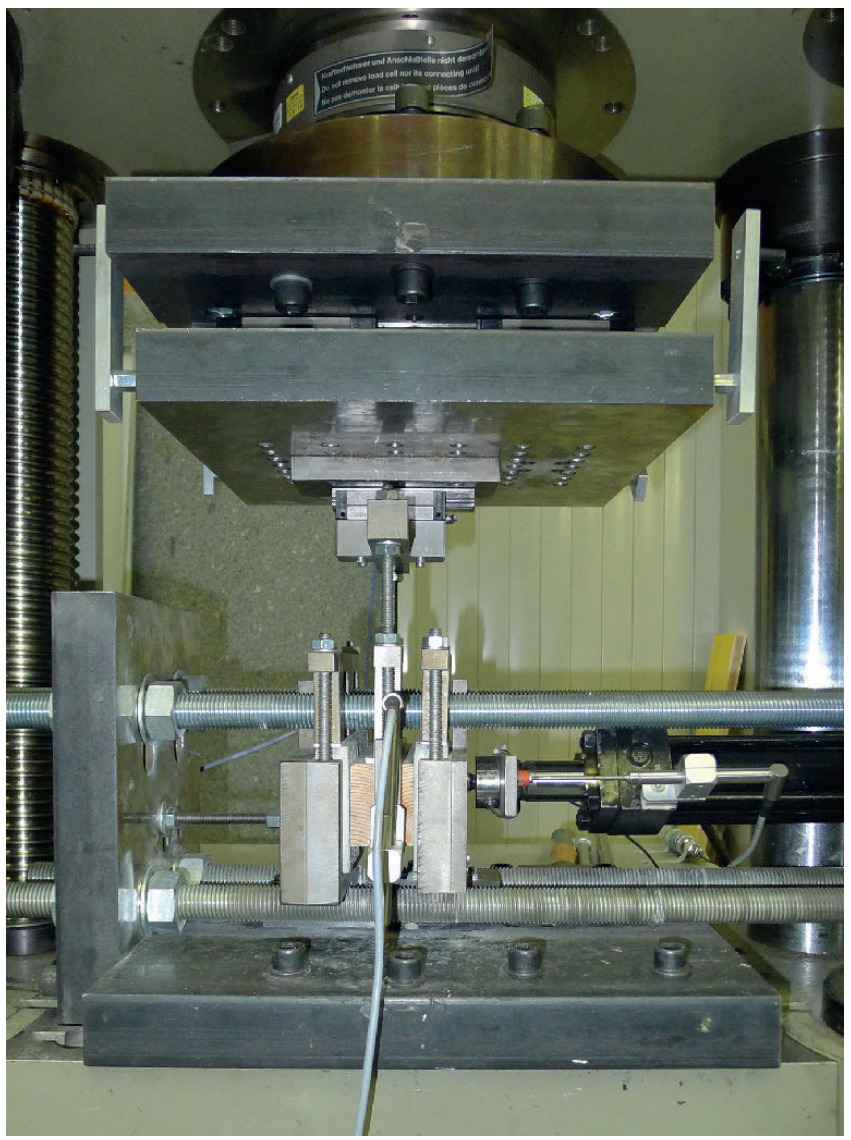

Bild 8. Testmaschine für zyklische Reibungsversuche an Holz-Holz- und Holz-Mörtel-Versuchskörpern [5] Abb.: T. Wenk

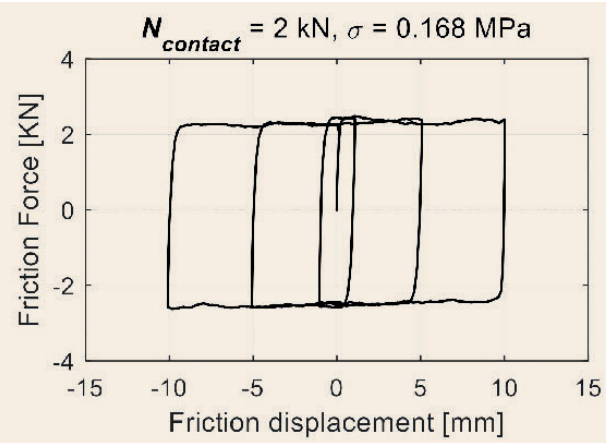

Bild 9. Kraft-Verschiebungs-Hysteresekurven eines Versuchskörpers aus modernem gehobeltem Holz in Kontakt mit einer Mörtelschicht [5] Abb.: J. Pacheco de Almeida gen hatten die Variation des Kontaktdrucks, der Belastungsgeschwindigkeit, der zyklischen Beanspruchung sowie der Faserrichtung des Holzes praktisch keinen Einfluss auf die Gleitreibungsbeiwerte [5].

\section{7 Überprüfungswerte der Gleitreibungsbeiwerte}

Für eine normgemässe Überprüfung werden sogenannte Überprüfungswerte der Gleitreibungsbeiwerte $\mu_{\mathrm{d} \text {,act }}$ erforderlich, die ungünstige Abweichungen vom charakteristischen Wert $\mu_{\mathrm{k}}$ berücksichtigen [8]. $\mathrm{Zu}$ deren Bestimmung kann analog $\mathrm{zu}$ den Festlegungen der Bemessungswerte der Gleitreibungsbeiwerte von Lagern unter Erdbebeneinwirkung vorgegangen werden. In [9] werden dazu Teilsicherheitsbeiwerte von $\gamma_{\mathrm{m}}=1,2$ für Stahl auf Beton und von $\gamma_{\mathrm{m}}=2,0$ für Stahl auf Stahl festgelegt. Für die Gleitreibungsbeiwerte von Holz auf Holz sowie Holz auf Mörtel wird ein Teilsicherheitsbeiwert von $\gamma_{\mathrm{m}}=1,5$ empfohlen, der im mittleren Bereich der Werte in [9] für Stahl und Beton liegt.

Die Überprüfungswerte des Gleitreibungsbeiwerts berechnen sich somit $\mathrm{zu} \mu_{\mathrm{d}, \mathrm{act}}=\mu_{\mathrm{k}} / \gamma_{\mathrm{m}}$. Bei Rundung von $\mu_{\mathrm{d} \text {,act }}$ auf eine Kommastelle ergeben sich in Tabelle 3 nur noch drei unterschiedliche Werte für die sechs Oberflächenbedingungen in Tabelle 2, nämlich $\mu_{\text {d,act }}=0,4$ für Holz auf Mörtel unabhängig von der Art und der Oberflächenbehandlung des Holzes, $\mu_{\mathrm{d}, \text { act }}=0,3$ für gesägtes oder gehacktes Holz aufeinander sowie nur $\mu_{\mathrm{d}, \text { act }}=0,1$ für gehobeltes Holz aufeinander.

Die horizontale Reibungskraft wird mit dem kleinsten Überprüfungswert der Normalkraft zugehörig zur betrachteten horizontalen Erdbebeneinwirkung bestimmt. Dabei sollte auch die vertikale Erdbebenanregung berücksichtigt werden.

\section{Modellierung der Holzbalkendecken}

Wichtig bei der Modellierung der Holzbalkendecken ist, dass die Flexibilität in ihrer Ebene berücksichtigt wird. Bei einer Modellierung mit starren Decken könnten nämlich die Beanspruchungen in den Decken, in den Verbindungen zur Wand und in aussen liegenden Wänden stark überschätzt werden. Erfolgt die Berechnung mit einer Modellierung mit flexiblen Decken, dann können die Wände mittels verformungsbasierter Nachweisen meist im Istzustand als genügend erdbebensicher akzeptiert werden [10]. Da der Verschiebungsbedarf der Antwortspektren in der revidierten Norm SIA 261 tendenziell kleiner wird, gewinnen verformungsbasierte Nachweise in Zukunft noch weiter an Bedeutung [11].

Bei der Modellierung der Verbindungen zwischen Decken und Wänden sollte eine gewisse Nachgiebigkeit der Verbindungen be-

Tabelle 2. Gleitreibungsbeiwerte für verschiedene Oberflächenbedingungen gemittelt aus 187 Versuchen.

\begin{tabular}{|c|c|c|c|c|c|}
\hline Gleitreibungsbeiwert & $\begin{array}{c}\text { gehobeltes } \\
\text { modernes Holz auf } \\
\text { Mörtel }\end{array}$ & $\begin{array}{c}\text { gesägtes modernes } \\
\text { Holz auf Mörtel }\end{array}$ & $\begin{array}{c}\text { gehacktes antikes } \\
\text { Holz auf Mörtel }\end{array}$ & $\begin{array}{c}\text { gehobeltes } \\
\text { modernes Holz } \\
\text { aufeinander }\end{array}$ & $\begin{array}{c}\text { gesägtes modernes } \\
\text { Holz aufeinander } \\
\text { Holz aufeinander }\end{array}$ \\
\hline Mittelwert $\mu$ & 0,64 & 0,77 & 0,70 & 0,29 \\
\hline $\begin{array}{c}\text { Standard- } \\
\text { abweichung }\end{array}$ & 0,07 & 0,08 & 0,09 & 0,08 \\
\hline $\begin{array}{c}\text { charakteristischer } \\
\text { Wert } \mu_{\mathrm{k}}\end{array}$ & 0,52 & 0,64 & 0,56 & 0,09 \\
\hline
\end{tabular}


Tabelle 3. Überprüfungswerte der Gleitreibungsbeiwerte für verschiedene Oberflächenbedingungen

\begin{tabular}{|c|c|c|c|}
\hline Gleitreibungsbeiwert & $\begin{array}{c}\text { Holz auf } \\
\text { Mörtel }\end{array}$ & $\begin{array}{c}\text { gesägtes oder } \\
\text { gehacktes Holz } \\
\text { aufeinander }\end{array}$ & $\begin{array}{c}\text { gehobeltes } \\
\text { Holz } \\
\text { aufeinander }\end{array}$ \\
\hline Teilsicherheitsbeiwert $\gamma_{\mathrm{m}}$ & 1,5 & 1,5 & 1,5 \\
\hline Überprüfungswert $\mu_{\mathrm{d}, \text { act }}$ & 0,4 & 0,3 & 0,1 \\
\hline
\end{tabular}

rücksichtigt werden. In Berechnungsprogrammen werden Verbindungen ohne spezielle Modellierung oft als starr angenommen und deren Kräfte werden in den Resultaten nicht aufgeführt. Dies bedeutet jedoch a priori nicht, dass sie den Anforderungen genügen würden.

\section{Ertüchtigungsstrategien}

Die effizienteste Ertüchtigungsstrategie ist keine Ertüchtigung, wenn also der Ist-Zustand dank ohne Massnahmen akzeptiert werden kann. Dies gilt im besonderen Masse für denkmalgeschützte Bauten. Die primäre Ertüchtigungsstrategie ist die Verstärkung der Verbindungen zwischen Decken und Wänden mit Ankern, soweit die Reibungskräfte nicht ausreichen. Sekundär sollte die Steifigkeit und die Tragfähigkeit der Decke in ihrer Ebene, zum Beispiel mit einer Beplankung der Balkenlage mit Holzwerkstoffplatten, soweit erhöht werden, damit sichergestellt ist, dass einerseits das Gebäude als Ganzes zusammenwirkt, um die Erdbebenbeanspruchungen abzutragen, und andererseits die Wände quer zur Wandebene gehalten sind.

\section{Zusammenfassung}

Bei der Überprüfung bezüglich Erdbeben von Holzbalkendecken in Bestandesbauten sind oft die Verbindungen zwischen Decken und Wänden die kritischen oder sogar fehlenden Bauteile. Für Bauten in niedriger bis mittlerer Seismizität genügen oft die Reibungskräfte als Verbindungen, doch fehlten bisher Angaben zu den Reibungsbeiwerten. Im vorliegenden Beitrag werden Überprüfungswerte der Gleitreibungsbeiwerte für Holz-Holz- und Holz-Mörtel-Verbindungen vorgestellt, die in der Praxis für die Überprüfung von Gebäuden mit Holzbalkendecken verwendet werden können. Die Gleitreibungsbeiwerte basieren auf der Auswertung von 187 statisch-zyklischen Versuchen. Mit diesen Gleitreibungsbeiwerten kann eine wichtige Lücke bei den Nachweisen von Gebäuden mit Holzbalkendecken bezüglich Erdbeben geschlossen werden.

\section{DANKSAGUNG}

Dieses Projekt wurde realisiert mit Unterstützung des schweizerischen Bundesamts für Umwelt (BAFU) im Rahmen des Aktionsplans Holz.
Literatur

[1] Beyer, K.; Penna, A.; Wenk, T.: Erdbebenüberprüfung von Natursteinmauerwerksgebäuden - das Basel-Projekt. In: Zabel, V.; Beinersdorf S. (Hrsg.): Vortragsband der 15. D-A-CH-Tagung Erdbebeningenieurwesen und Baudynamik. Weimar, 2017.

[2] Bundesamt für Statistik (BFS): Gebäude- und Wohnungsstatistik 2018 , www.bfs.admin.ch/bfs/de/home/statistiken/bau-wohnungswesen/ge baeude.gnpdetail.2019-0315.html [Zugriff am: 04.03.2020].

[3] Fäh, D.; Gisler, M.; Jaggi, B. et al.:The 1356 Basel Earthquake: an Interdisciplinary Revision. In: Geophysical Journal International, Vol. 178 (2009), Iss. 1, pp. 351-374.

[4] Norm SIA 269/8: Erhaltung von Tragwerken - Erdbeben. Schweizerischer Ingenieur- und Architekten-Verein, Zürich 2017.

[5] Pacheco de Almeida, J.; Beyer, K.; Brunner R. et al.: Überprüfung und Ertüchtigung von Holzbalkendecken in Bestandesbauten bezüglich Erdbeben. Interim Report, Earthquake Engineering and Structural Dynamics Laboratory (EESD) École Polytechnique Fédérale de Lausanne (EPFL), Lausanne 2018.

[6] Pacheco de Almeida, J.; Beyer, K.; WenkT. et al.: Characterization of mortar-timber and timber-timber cyclic friction in timber floor connections of masonry building. Submitted for publication to Mater. and Struct., 2019.

[7] Steiger, R.; Fink, G.; Nerbano, S. et al.: Experimental investigation of friction stresses between adjacent panels made of Oriented Strand Board (OSB) and between OSB panels and glued laminated timber (GLT) frame members. In: Materials and Structures, Vol. 51 (2018), Iss. 1.

[8] Norm SIA 260: Grundlagen der Projektierung von Tragwerken. Schweizerischer Ingenieur- und Architekten-Verein, Zürich 2013.

[9] Bundesamt für Straßen: Richtlinien für konstruktive Einzelheiten von Brücken, Kapitel 1: Lager. ASTRA 12 004, Bern, 2011.

[10] Vanin, F; Zaganelli, D.; Penna, A. et al.: Estimates for the stiffness, strength and drift capacity of stone masonry walls based on 123 quasistatic cyclic tests reported in the literature. In: Bulletin of Earthquake Engineering, Vol. 15 (2017), pp. 5435-5479.

[11] Norm FprSIA 261:2020: Einwirkung aufTragwerke, Einspracheverfahren, Schlussentwurf des Kapitels 16 Erdbeben. Schweizerischer Ingenieur- und Architektenverein, Zürich, 2020.

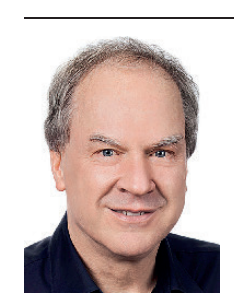

Dr. sc. techn. Thom a s We n k

Gehrenholz 2h, 8055 Zürich, Schweiz

Abb.: S. Baumgartner

Ro I a n $\mathrm{d}$ r u n n e r

Lignum, 8008 Zürich, Schweiz

Prof. D r.

J o ão Pacheco de A I meid a

Université catholique de Louvain (UCLouvain) Institute of Mechanics, Materials and Civil Engineering (iMMC)

1348 Louvain-la-Neuve, Belgien

Prof. Dr. Katrin B y e r

Ecole Polytechnique Fédérale de Lausanne

(EPFL)Earthquake Engineering and

Structural Dynamics Laboratory (EESD)

ENAC

1015 Lausanne, Schweiz 